\title{
Corrigendum to "A Diagnosis Method for Rotation Machinery Faults Based on Dimensionless Indexes Combined with $K$-Nearest Neighbor Algorithm"
}

\author{
Jianbin Xiong, ${ }^{1,2}$ Qinghua Zhang, ${ }^{1,2}$ Zhiping Peng, ${ }^{2}$ Guoxi Sun, \\ Weichao $\mathrm{Xu},{ }^{3}$ and Qi Wang ${ }^{2}$ \\ ${ }^{1}$ Guangdong Provincial Key Laboratory of Petrochemical Equipment Fault Diagnosis, Maoming 525000, China \\ ${ }^{2}$ School of Computer and Electronic Information, Guangdong University of Petrochemical Technology, Maoming 525000, China \\ ${ }^{3}$ School of Automation, Guangdong University of Technology, Guangzhou 510006, China
}

Correspondence should be addressed to Guoxi Sun; 158011382@qq.com

Received 3 January 2016; Accepted 13 December 2016; Published 28 June 2017

Copyright (C) 2017 Jianbin Xiong et al. This is an open access article distributed under the Creative Commons Attribution License, which permits unrestricted use, distribution, and reproduction in any medium, provided the original work is properly cited.

In the article titled "A Diagnosis Method for Rotation Machinery Faults Based on Dimensionless Indexes Combined with K-Nearest Neighbor Algorithm" [1], there was an error in the "Theory" section which should be corrected as follows.

(4) when $T=\infty, m=4$, kurtosis index $K_{v}$ is defined as

$$
K_{v}=\lim _{T \rightarrow \infty} \frac{\left[\int_{-\infty}^{+\infty}|x|^{4} \xi(x) d x\right]}{\left[\int_{-\infty}^{+\infty} x^{2} \xi(x) d x\right]^{2}}=\frac{E\left(|x|^{4}\right)}{\left[E\left(|z|^{2}\right)\right]^{2}}
$$

should be corrected to

(4) when $T=4, m=2$, kurtosis index $K_{v}$ is defined as

$$
K_{v}=\lim _{T \rightarrow \infty} \frac{\left[\int_{-\infty}^{+\infty}|x|^{4} \xi(x) d x\right]^{1 / 4}}{\left[\int_{-\infty}^{+\infty} x^{2} \xi(x) d x\right]^{1 / 2}}=\frac{E\left(|x|^{4}\right)}{\left[E\left(|z|^{2}\right)\right]^{2}} .
$$

\section{References}

[1] J. Xiong, Q. Zhang, Z. Peng, G. Sun, W. Xu, and Q. Wang, "A diagnosis method for rotation machinery faults based on dimensionless indexes combined with $K$-nearest neighbor algorithm," Mathematical Problems in Engineering, vol. 2015, Article ID 563954, 9 pages, 2015. 


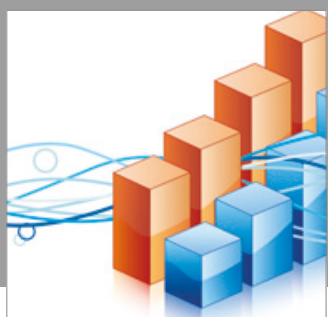

Advances in

Operations Research

vatersals

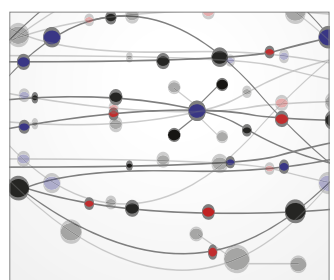

\section{The Scientific} World Journal
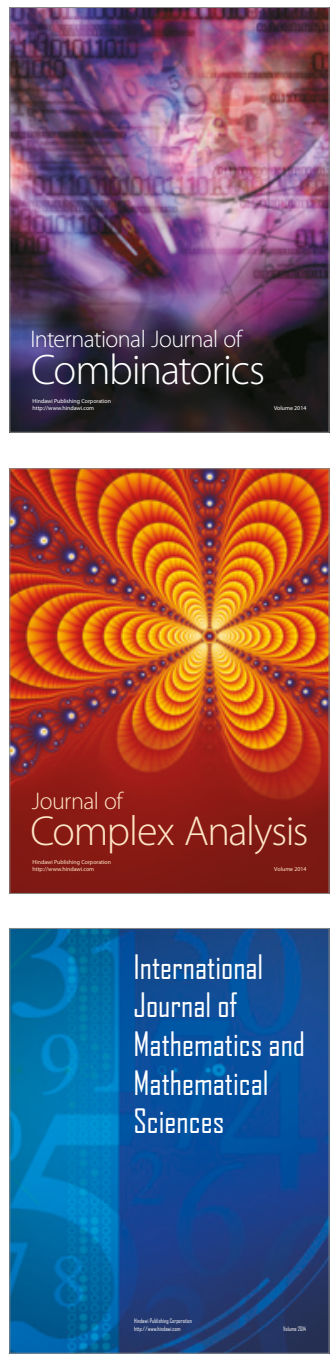
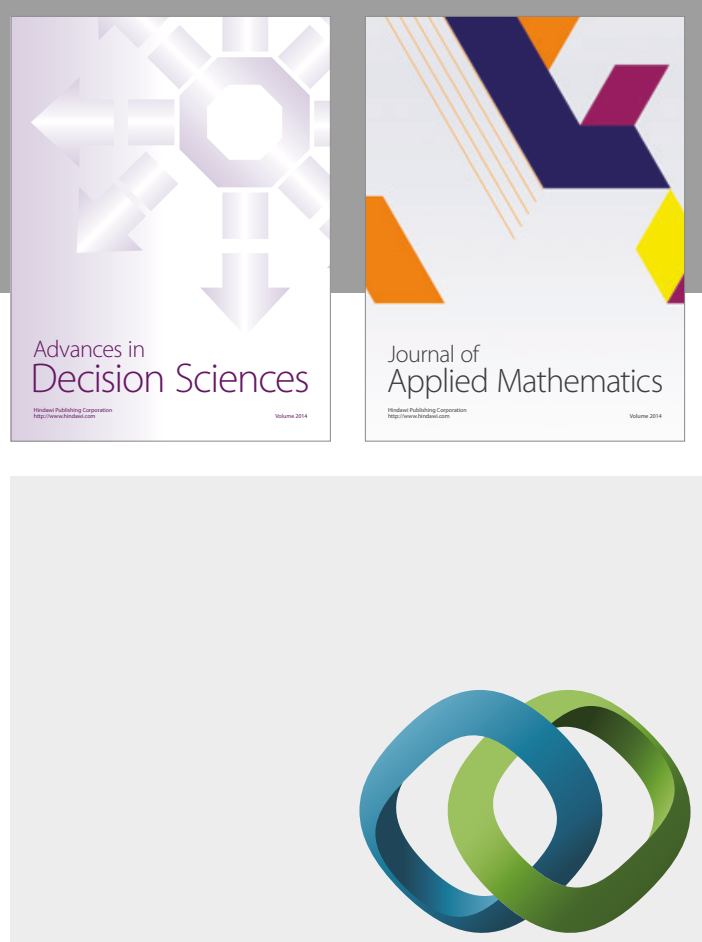

\section{Hindawi}

Submit your manuscripts at

https://www.hindawi.com
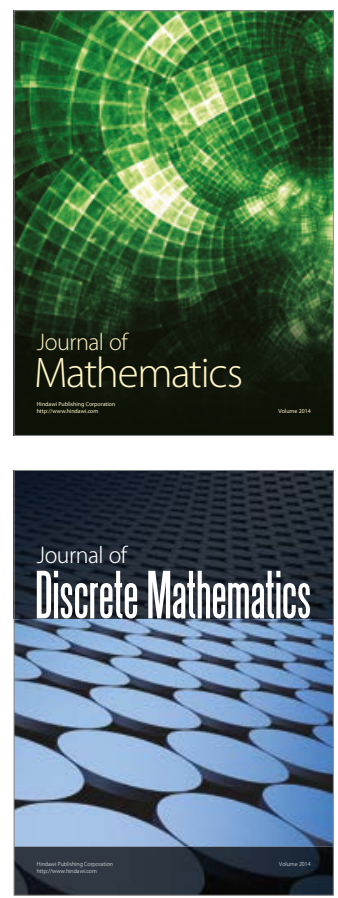

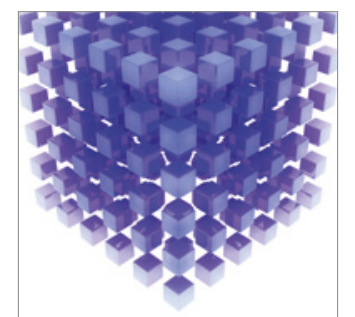

Mathematical Problems in Engineering
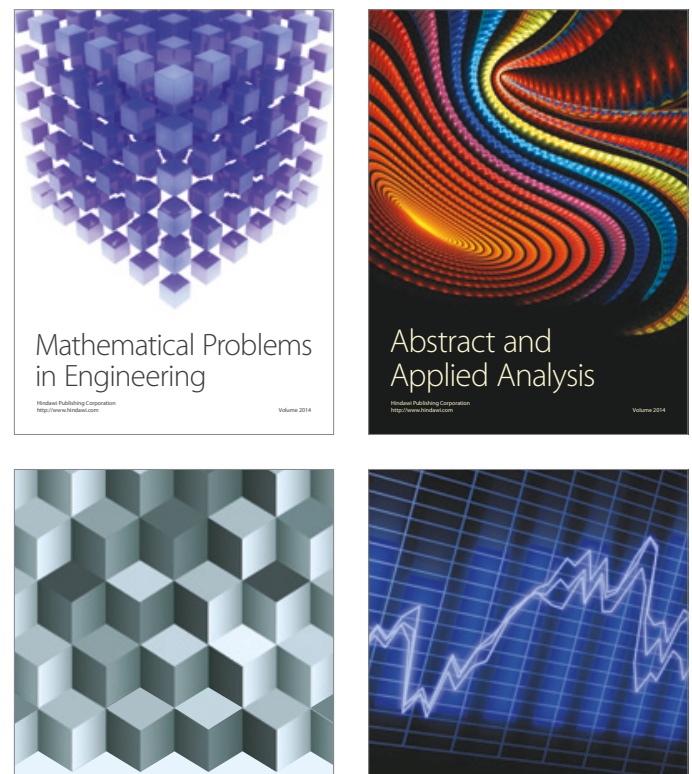

Journal of

Function Spaces

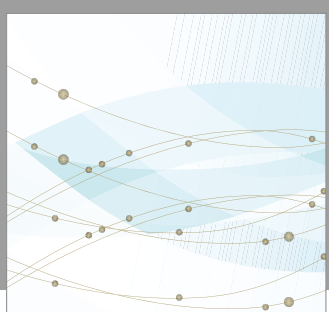

Algebra

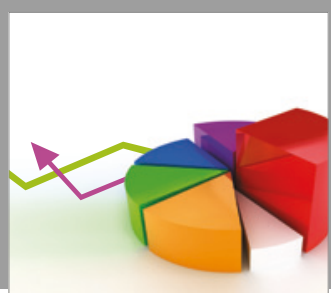

Journal of

Probability and Statistics

\section{vatemesion}

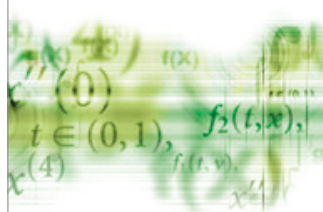

International Journal of

Differential Equations
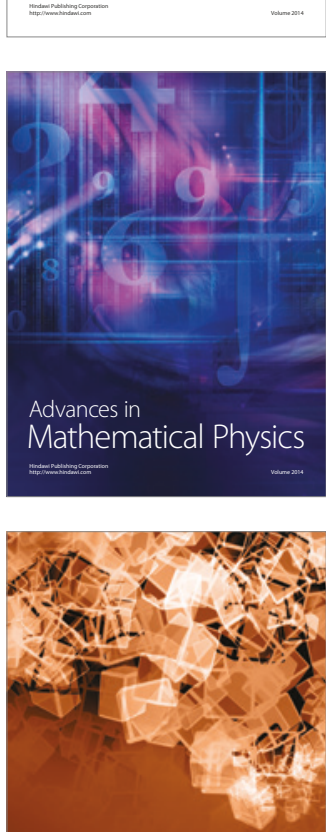

Discrete Dynamics in Nature and Society
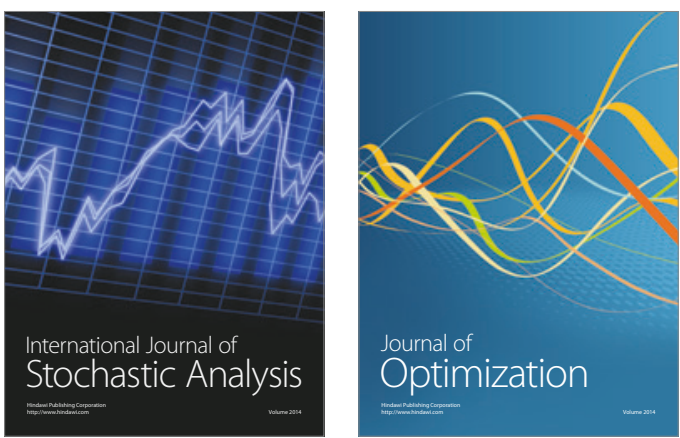